\title{
Diffraction of plane wave on a periodic layer with arbitrary permittivity distribution *
}

\author{
Dmitri Knyazkov
}

\author{
A.Ishlinsky Institute for Problems in Mechanics of the Russian \\ Academy of Sciences, Moscow, Russia (e-mail: knyaz@ipmnet.ru)
}

Keywords: Diffraction, scattering, method of projections, sea surface radiometry

\section{INTRODUCTION}

A problem of modeling diffraction of a plane electromagnetic wave on a layer is considered. The layer is periodic by two directions and is bounded by two surfaces along the third one. These surfaces are also periodic and can have any form along those two directions. The permittivity of the layer is given by an arbitrary function. This function can even be discontinuous. Thus, in the framework of this model, a number of diffraction problems can be solved, e.g. diffraction on a grating or on a hologram, diffraction on earth or sea surfaces, problems of propagation of an electromagnetic wave in a waveguide, etc.

It is needed to calculate the diffracted electromagnetic field. The reflected and the propagated through the layer fields should be found. Coefficients of reflection, transmission and absorption (in the case of an absorbing medium) should also be computed. Therefore, in the described formulation, the following direct and inverse (see Kirsch (2011)) problems of diffraction can be solved:

- the tomography or the holography, where it is necessary to calculate the wave that passed through the media, or to synthesize a hologram with desired properties;

- the inverse scattering or the tomography of the inside of the layer, that requres to find the reflected from layer wave, for example, detection of underground objects as in Fiaz et al. (2012), or identifying the layer surface shape, for example, the active radar probing of the ocean surface;

- the determination of a shape of a layer based on information about its self-radiation, for example, the passive radio probing of the sea surface as in Gavrikov et al. (2016), where it is necessary to calculate the absorption coefficient.

The latter is possible thanks to the reciprocity principle, see Tsang et al. (2000). According to this principle, the self-radiation of a medium in a given direction is in the proportion to an energy absorbed by the medium under irradiation by a plane wave from this direction.

Solution of the outlined above problem of diffraction of the wave on the layer was described earlier for the two dimensional cases of H-polarization and E-polarization (see

\footnotetext{
* The reported study was funded by RFBR, according to the research project No. 16-31-60096 mol-a-dk.
}

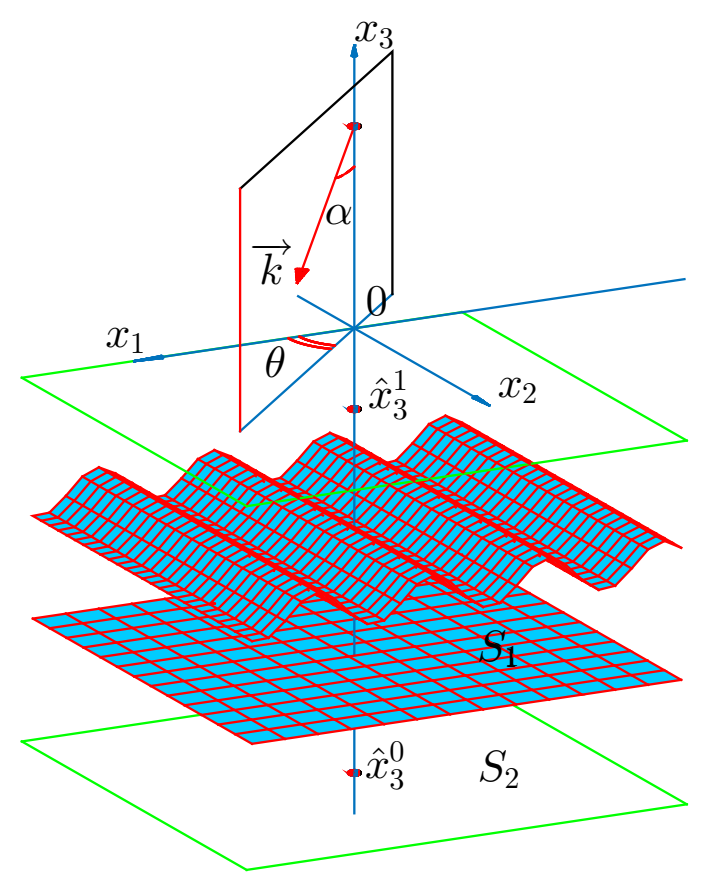

Fig. 1. Scheme of the wave incidence

Knyazkov (2017b,a)). In the current paper, the substantially three dimensional case is considered. The method of projections proposed in Il'inskiy (1998) is used to calculate the result of diffraction. This approach allows us to reduce the original problem to a system of ordinary differential equations (ODEs) of the first order.

\section{PROBLEM STATEMENT}

The wave comes at arbitrary inclination to the layer's surface. Its wave-vector $\vec{k}$ has the angle $\alpha$ with the axis $O x_{3}$ (see Fig. 1). The projection of this vector to the $O x_{1} x_{2}$ plane forms the angle $\theta$ with the $O x_{1}$ axis. The layer is bounded by two surfaces defined by the functions $S_{1}\left(x_{1}, x_{2}\right)$ and $S_{2}\left(x_{1}, x_{2}\right)$. The permittivity of the layer is given by the function $\varepsilon\left(x_{1}, x_{2}, x_{3}\right)$. Functions $S_{1}, S_{2}, \varepsilon$ are periodic along $O x_{1}$ and $O x_{2}$ axes with corresponding periods $a_{1}$ and $a_{2}$.

The Maxwells equations for the case of time-harmonic waves can be used. In the three dimensional space they lead to the following system for the components of the 
complex phasor amplitude electric field intensity vector $\vec{E}=\left(E_{1}, E_{2}, E_{3}\right)$ :

$$
\left\{\begin{array}{l}
E_{1,22}+E_{1,33}-E_{3,13}-E_{2,12}+\varepsilon k_{0}^{2} E_{1}=0 \\
E_{2,11}+E_{2,33}-E_{1,21}-E_{3,23}+\varepsilon k_{0}^{2} E_{2}=0 \\
E_{3,11}+E_{3,22}-E_{1,31}-E_{2,32}+\varepsilon k_{0}^{2} E_{3}=0,
\end{array}\right.
$$

where $E_{i, j k}=\frac{\partial}{\partial x_{k}} \frac{\partial}{\partial x_{j}} E_{i}\left(x_{1}, x_{2}, x_{3}\right)$. The goal is to solve the problem (1) with the corresponding radiation conditions in the domain $\Omega=\left[0, a_{1}\right] \times\left[0, a_{2}\right] \times\left[\hat{x}_{3}^{0}, \hat{x}_{3}^{1}\right]$.

\section{THE METHOD OF PROJECTIONS}

The system (1) can be solved with the use of the method of projections proposed in Il'inskiy (1998). The vector function $\vec{E}\left(x_{1}, x_{2}, x_{3}\right)$ can be represented as

$$
E_{i}\left(x_{1}, x_{2}, x_{3}\right)=\sum_{s=1}^{N} B_{i s}\left(x_{3}\right) \psi_{s}\left(x_{1}, x_{2}\right), \quad i=1,2,3 .
$$

Here, functions $\psi_{s}$ have the following property

$$
\int_{0}^{a_{2}} \int_{0}^{a_{1}} \psi_{s_{1}}\left(x_{1}, x_{2}\right) \psi_{s_{2}}^{*}\left(x_{1}, x_{2}\right) d x_{1} d x_{2}=\delta_{s_{1}, s_{2}},
$$

where $s_{1}, s_{2}=1, \ldots, N, f^{*}$ is the conjugate of $f, \delta_{i j}$ is Kronecker delta, $\delta_{i j}=1$ for $i=j, \delta_{i j}=0$ for $i \neq j$.

After substituting the solution expansion (2) to the equations from (1), multiplying them by $\psi_{s}^{*}\left(x_{1}, x_{2}\right), s=$ $1, \ldots, N$ and integrating on $\left[0, a_{1}\right] \times\left[0, a_{2}\right]$ we will have the following system of $4 N$ first order ODEs:

$$
\left\{\begin{array}{l}
p^{\prime}=Q b, \\
b^{\prime}=C p,
\end{array}\right.
$$

where $Q$ and $C$ are some matrixes with piece-wise continuous coefficients, $p$ and $b$ are the vectors of unknown variables. The boundary conditions can be written as

$$
\left\{\begin{array}{l}
C p+\left.i G b\right|_{x_{3}=\hat{x}_{3}^{0}}=0, \\
C p-\left.i G b\right|_{x_{3}=\hat{x}_{3}^{1}}=v,
\end{array}\right.
$$

where $G$ is the diagonal matrix and the vector $v$ is defined by the initial wave:

$$
\begin{aligned}
v= & \left(v_{1}, \ldots, v_{n}, \ldots, v_{2 N}\right)^{T} \\
= & \left(-2 i \gamma_{1} \sqrt{a_{1} a_{2}} E_{01} e^{-i \gamma_{1} \hat{x}_{3}^{1}} \delta_{n, 1}, 0\right. \\
& \left.\ldots, 0,-2 i \gamma_{1} \sqrt{a_{1} a_{2}} E_{02} e^{-i \gamma_{1} \hat{x}_{3}^{1}} \delta_{n, N+1}, 0, \ldots, 0\right)^{T}
\end{aligned}
$$

where $\gamma_{1}=|\vec{k}| \cdot \cos (\alpha)$.

After solving the resulting system of ODEs (4) with the boundary conditions (5), we obtain the functions $B_{n s}, n=$ $1,2,3, s=1, \ldots, N$. Substitution of these function to (2) gives us the desired solution. When knowing the solution, the reflection, transmission and absorption coefficients could easily be calculated.

\section{COMPUTATIONAL PROGRAM}

The computer program is written, that implements the described procedure. It is verified by comparison with an analytical solution in the simple cases of an oblique incidence on a homogeneous plane layer. In the other series of computations, the results are compared with the results obtained by another program for the case of $\mathrm{H}$-polarized or E-polarized wave and homogeneous cylindrical layer with a sinusoidal surface (see Knyazkov (2017b,a)). Numerical experiments show the high accuracy. The error is less than $0.1 \%$ for computational meshes, that has 200-300 points along each of the coordinate axes. Some calculations for the needs of sea surface radiometry are performed. The good sensitivity and good selectivity to sea surface disturbances is shown for the inverse problem of sea surface form identification.

\section{CONCLUSIONS AND OUTLOOK}

In this paper, the diffraction of plane wave on a periodic layer is considered. The layer has arbitrary permittivity distribution and is bounded by two surfaces, that have arbitrary shape. The method of projections is used which allows to reduce the initial problem to the system of first order ordinary differential equations.

The developed computer program can be used in the mentioned applications. To decrease calculation time, the program can be rewritten for the hybrid cluster architecture. The described above programs are available free of charge on the internet. The source code can be downloaded from https://bitbucket.org/Jclash/dpproj. Small calculations can be performed with the use of web-interface http://ipmnet.ru/ knyaz/diffraction.html (see Knyazkov (2017c)) at Hybrilit HPC cluster installed at the Laboratory of Information Technologies of the Joint Institute for Nuclear Research, Dubna, Russia.

\section{REFERENCES}

Fiaz, M.A., Frezza, F., Pajewski, L., Ponti, C., and Schettini, G. (2012). Scattering by a circular cylinder buried under a slightly rough surface: the cylindrical-wave approach. IEEE Transactions on antennas and propagation, 60(6), 2834-2842.

Gavrikov, A., Knyazkov, D., Romanova, A., Chernik, V., and Shamaev, A. (2016). Simulation of influence of the surface disturbance on the ocean self radiation spectrum. Program systems: theory and applications, 7(2), 73-84. doi:10.25209/2079-3316-2016-7-2-73-84.

Il'inskiy, A.S. (1998). A method of investigating wave diffraction problems on a periodic structure. USSR Computational Mathematics and Mathematical Physics, 14(4), 242-246.

Kirsch, A. (2011). An Introduction to the Mathematical Theory of Inverse Problems, volume 120 of Applied Mathematical Sciences. Springer, 2nd edition edition.

Knyazkov, D. (2017a). Simulating diffraction of plane wave on periodic layer with the use of the method of projections. IEEE Proc. of Days on Diffraction 2017, Saint-Petersburg, Russia, 180-185. doi: 10.1109/DD.2017.8168019.

Knyazkov, D. (2017b). Simulation of diffraction on a layer using the method of projections. AIP Conference Proceedings, 1863, 370006. doi:10.1063/1.4992553.

Knyazkov, D. (2017c). Web-interface for hpc computation of a plane wave diffraction on a periodic layer. Lobachevskii Journal of Mathematics, 38(5), 936-939. doi:10.1063/1.4992553.

Tsang, L., Kong, J.A., and Ding, K.H. (2000). Scattering of Electromagnetic Waves, volume Vol. 1: Theory and Applications. Wiley Interscience. 